\title{
Measuring Consumer Expectation in Banking Sector: A Comparative Analysis"
}

\author{
Dr. Shilpi Gupta \\ Assistant Professor, Department of Economics, Central University of Rajasthan
}

\begin{abstract}
This study is intended to compare the expectations of Indian consumers from their banks with what bank actually provides. Additionally, the study helps to determine if there is any significant difference in consumers' expectations and banks' offerings between public sector and private sector banks in India. This paper reports on a quantitative study of executives working in private and public sector organizations and colleges in Northern India. The objective of this study is to determine the factors that have the significant gap between expectations and actual in terms of banks' service quality. There is not any significant difference between consumers' expectations between private and public sector banks. There is significant difference between private and public sector banks' actual offerings. There is significant difference (except Tangible factor) between consumers' expectations and bank's actual offerings for Private sector banks. There is significant difference between consumers' expectations and bank's actual offerings for Public sector banks.

The deviation of consumers' expectations and bank's actual offerings is more in public sector banks as compared to private sector banks.
\end{abstract}

\section{Introduction:}

Banking Industry is the backbone of Indian economy. Indian banking industry is major divided into two sectors - public sector banks and private sector banks. The public sector banks have the major players like $\mathrm{SBI}$, PNB, and OBC etc while the private sector is dominated by ICICI, HDFC, Citibank, Standard Charted etc. In this competitive world, it has become very important for the banks to not only introduce the new marketing concepts but also to bring satisfaction to their customers. It is reported that $90 \%$ of bank switching in the Asian banking sector occurs due to pricing, service quality and inconvenience (Gerrard and Cunningham, 2004). Hence it is important for the banks to determine the factors that the consumers' perceive as missing in banks' offerings and try to bridge these gaps.

This study aims to compare customers expectations and banks' actual offerings for both private and public sector banks individually and then compare them to determine the factors having the significant gap. The banks' should improve these factors to retain/acquire the existing/new customers.

\section{Based upon above discussion, we hypothesize the following -}

H1: There is no significant difference between customer expectations and customer perception of private banks regarding service quality.

$\mathrm{H} 2$ : There is no significant difference between customer expectations and customer perception of public banks regarding service quality.

H3: There is no significant difference between the two groups of banks with respect to the expectations of the customers.

H4: There is no significant difference between the two groups of banks with respect to the customers' perception.

\section{Participants and procedure-}

\section{Research Methodology:}

The data for this study is collected in March, 2010 and consisted of 121 respondents, major working as middle management executives in private and public sector organizations in Northern India. The survey is also conducted on the lecturers of one of the reputed engineering and Management College in Gurgaon. The participants are chosen randomly across the organizations. Likert scale is chosen to answer the questionnaire. The same consist of both male and female participants.

\section{Measures-}

Following twenty variables are chosen initially to survey the consumers -

Your bank should have convenient branch locations/ATMs Employee's behavior

The behavior of employees of your bank develops confidence in customers. Online banking facility 
Employees of your bank should be consistently courteous with you.

Your bank should have online banking facility.

Your bank should provide its services at the time it promises to do so.

Your bank should have operating hours convenient to all its customers.

When you have a problem, your bank should show a sincere interest in solving it.

Your bank should charge you extra penny for online services.

Your bank should perform the service right the "first" time.

Employees of your bank should tell you exactly when services will be performed.

Your bank's physical facilities should be visually appealing.

Your bank's employees should be neat - appearing.

Employees of your bank should have the knowledge to answer your questions.

Your bank should update you about the new product or interest rate on your mail id or mobile.

Your bank should give you individual attention.

Materials associated with the service, such as pamphlets and statements should be visually appealing at your bank.

Employees of your bank should be always willing to help you

When your bank promises to do something by a certain time, it should do so.

Your bank should have modern-looking equipment

You should feel safe in your transactions with your bank.

All the above variables are rated on a 6-point Likert scale ranging from 1(Strongly disagree) to 6(Strongly Agree). The factor analysis of above twenty variables is performed through SPSS tool. Brief description of factors and their mapping with the variables is present in Results section below.

\section{Factor Analysis:}

\section{Results and Discussions:}

Following is the result of factor analysis performed on the data collected of twenty variables based upon consumers' expectations.

\begin{tabular}{|c|c|c|c|c|c|c|c|c|c|}
\hline \multirow[b]{3}{*}{ Component } & \multicolumn{9}{|c|}{ Total Variance Explained } \\
\hline & \multicolumn{3}{|c|}{ Initial Eigenvalues } & \multicolumn{3}{|c|}{ Extraction Sums of Squared Loadings } & \multicolumn{3}{|c|}{ Rotation Sums of Squared Loadings } \\
\hline & Total & $\%$ of Variance & Cumulative $\%$ & Total & $\%$ of Variance & Cumulative \% & & $\%$ of Variance & Cumulative \% \\
\hline & 4.574 & 22.872 & 22.872 & 4.574 & 22.872 & 22.872 & 2.566 & 12.831 & 12.831 \\
\hline 2 & 2.055 & 10.275 & 33.147 & 2.055 & 10.275 & 33.147 & 2.293 & 11.465 & 24.296 \\
\hline 3 & 1.494 & 7.469 & 40.616 & 1.494 & 7.469 & 40.616 & 2.122 & 10.610 & 34.906 \\
\hline 4 & 1.264 & 6.319 & 46.935 & 1.264 & 6.319 & 46.935 & 1.728 & 8.642 & 43.548 \\
\hline 5 & 1.195 & 5.973 & 52.909 & 1.195 & 5.973 & 52.909 & 1.479 & 7.396 & 50.944 \\
\hline 6 & 1.189 & 5.944 & 58.853 & 1.189 & 5.944 & 58.853 & 1.311 & 6.555 & 57.499 \\
\hline 7 & 1.038 & 5.189 & 64.041 & 1.038 & 5.189 & 64.041 & 1.309 & 6.543 & 64.041 \\
\hline 8 & .933 & 4.664 & 68.705 & & & & & & \\
\hline 9 & .901 & 4.507 & 73.212 & & & & & & \\
\hline 10 & .789 & 3.944 & 77.156 & & & & & & \\
\hline 11 & .756 & 3.778 & 80.934 & & & & & & \\
\hline 12 & .697 & 3.483 & 84.417 & & & & & & \\
\hline 13 & .580 & 2.902 & 87.319 & & & & & & \\
\hline 14 & .525 & 2.627 & 89.946 & & & & & & \\
\hline 15 & .456 & 2.278 & 92.224 & & & & & & \\
\hline 16 & .412 & 2.058 & 94.282 & & & & & & \\
\hline 17 & .346 & 1.731 & 96.014 & & & & & & \\
\hline 18 & .303 & 1.514 & 97.528 & & & & & & \\
\hline 19 & 264 & 1.322 & 98.850 & & & & & & \\
\hline 20 & .230 & 1.150 & 100.000 & & & & & & \\
\hline
\end{tabular}

Figure 1 - Factor Analysis

Rotated Component Matriz

\begin{tabular}{|c|c|c|c|c|c|c|c|}
\hline & \multicolumn{7}{|c|}{ Component } \\
\hline & 1 & 2 & 3 & 4 & 5 & 6 & \\
\hline 18. When y our bank promises to do something by a certain time, it should do so. & .818 & & & & & & \\
\hline 20. You should feel saf e in your transactions with your bank. & .713 & & & & & & \\
\hline 13. Employ ees of y our bank should have the knowledge to answer your questions. & .607 & & . 410 & & & & \\
\hline 10. Employ ees of y our bank should tell y ou exactly when services will be performed & .395 & & & & & & \\
\hline 12. Your bank's employees should be neat - appearing. & & 815 & & & & & \\
\hline 11. Your bank's physical facilities should be $v$ isually appealing. & & .762 & & & & & \\
\hline 19. Your bank should have modern-looking equipment. & & .724 & & & & & \\
\hline $\begin{array}{l}\text { 14. Your bank should update you about the new product or interest rate on y our mail } \\
\text { id or mobile. }\end{array}$ & & .504 & & & & & \\
\hline 6. Your bank should have operating hours conv enient to all its customers. & & & 665 & & & & \\
\hline 1. Your bank should have convenient branch locations/ATMs. & & & .654 & & & & \\
\hline 16. Materials associated with the serv ice, such as pamphlets and statements should & & & 568 & & & & \\
\hline $\begin{array}{l}\text { be visually appealing at your bank. } \\
5 \text { Your bank should provide its services at the time it promises to do so. }\end{array}$ & & & & & & & \\
\hline 4. Your bank should have online banking f acility. & 399 & & & & & & \\
\hline 7. When y ou have a problem, your bank should show a sincere interest in solv ing it. & & & .442 & 495 & & & \\
\hline $\begin{array}{l}\text { 2. The behavior of employees of your bank dev elops conf idence in customers. } \\
15 . \text { Your bank should give you individual attention. }\end{array}$ & & & & & $554>>2>3$ & & \\
\hline 8. Your bank should charge you extra penny for online serv ices. & & & & & & .876 & \\
\hline 9. Your bank should perf orm the serv ice right the "f irst" time. & & & & & & & \\
\hline
\end{tabular}

Extraction Method: Principal Component Analy sis.
Rotation Met hod: Varimax with Kaiser Normalization.

a. Rotation converged in 11 iterations.

Figure 2 - Factory - Variable Mapping

Following is the analysis of the above results - 
Twenty items were reduced to seven factor dimensions which explained $64.041 \%$ of the overall variance.

Following Seven factors identified with their mapping variables

\section{Responsiveness}

When your bank promises to do something by a certain time, it should do so.

Employees of your bank should tell you exactly when services will be performed.

You should feel safe in your transactions with your bank.

Employees of your bank should be always willing to help you

Employees of your bank should have the knowledge to answer your questions.

\section{Tangible}

Your bank's employees should be neat - appearing.

Your bank's physical facilities should be visually appealing.

Your bank should have modern-looking equipment

Your bank should update you about the new product or interest rate on your mail id or mobile.

\section{Empathy}

Your bank should have operating hours convenient to all its customers.

Your bank should have convenient branch locations/ATMs Employee's behavior

Employees of your bank should be consistently courteous with you.

Materials associated with the service, such as pamphlets and statements should be visually appealing at your bank.

\section{Reliability}

Your bank should provide its services at the time it promises to do so..

Your bank should have online banking facility.

When you have a problem, your bank should show a sincere interest in solving it

\section{Assurance}

The behavior of employees of your bank develops confidence in customers.

Your bank should give you individual attention.

\section{Cost to consumers}

Your bank should charge you extra penny for online services.

\section{Quality of Service}

Your bank should perform the service right the "first" time.

\section{Reliability Test}

\section{Reliability}

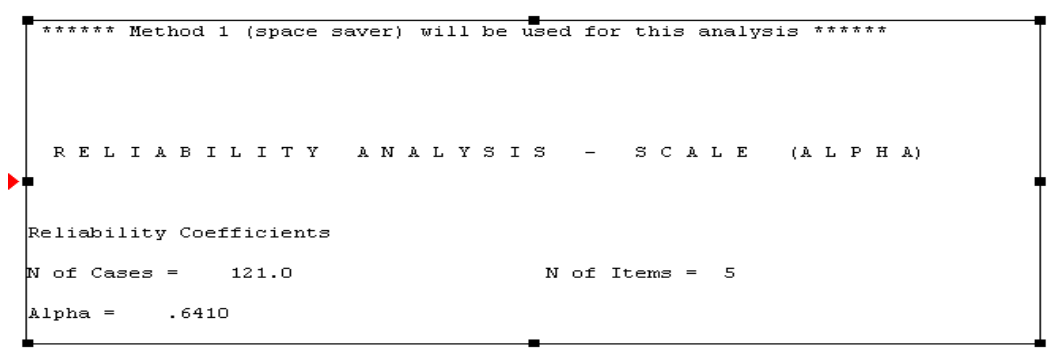

Figure 3 - Reliability Test

When the reliability test was performed on the above seven factors, the reliability was less than 0.6 hence last two factors (Cost to Consumers and Quality of Service) dropped to increase the reliability of the factors. Additionally, the two dropped factors have a single variable mapped hence not very significant.

\section{T-Test - Pvt. Banks Expectations vs. Actual}

\begin{tabular}{|ll|r|r|r|r|}
\hline \multicolumn{1}{|c|}{ Paired Samples Statistics } \\
\hline Pair 1 & Pvt. Responsiv eness Expect & Mean & N & Std. Dev iation & $\begin{array}{c}\text { St d. Error } \\
\text { Mean }\end{array}$ \\
& Pvt. Responsiv eness Actual & 5.5367 & 109 & .3658 & $3.504 \mathrm{E}-02$ \\
Pair 2 & Pvt. Tangible Expect & 4.7243 & 109 & .6852 & $6.563 \mathrm{E}-\mathrm{O} 2$ \\
& Pvt. Tangible Actual & 4.7943 & 109 & .6917 & $6.625 \mathrm{E}-\mathrm{O} 2$ \\
Pair 3 & Pvt. Empathy Expect & 5.1189 & 109 & .6097 & $5.840 \mathrm{E}-\mathrm{O} 2$ \\
& Pvt. Empathy Actual & 4.6280 & 110 & .5326 & $5.078 \mathrm{E}-\mathrm{O} 2$ \\
Pair 4 & Pvt. Reliability Expect & 5.6468 & 110 & .6466 & $6.166 \mathrm{E}-\mathrm{O} 2$ \\
& Pvt. Reliability Actual & 4.9664 & 109 & .4713 & $4.514 \mathrm{E}-\mathrm{O} 2$ \\
Pair 5 & Pvt. Assurance Expect & 5.1101 & 109 & .6510 & $6.235 \mathrm{E}-\mathrm{O} 2$ \\
& Pvt. Assurance Actual & 4.5183 & 109 & .6249 & $5.986 \mathrm{E}-\mathrm{O} 2$ \\
\hline
\end{tabular}

Figure 4 - Pvt. Banks' Expectations vs. Actual - Paired Samples Stats 


\begin{tabular}{|c|c|c|c|c|c|c|c|c|c|}
\hline \multicolumn{10}{|c|}{ Paired Samples Test } \\
\hline & & \multicolumn{5}{|c|}{ Paired Diff erences } & \multirow[b]{2}{*}{$t$} & \multirow[b]{2}{*}{$d f$} & \multirow[b]{2}{*}{ Sig. (2-tailed) } \\
\hline & & Mean & Std. Deviation & $\begin{array}{l}\text { Std. Error } \\
\text { Mean }\end{array}$ & Lower & Upper & & & \\
\hline Pair 1 & $\begin{array}{l}\text { Pvt. Responsiv eness Expect - } \\
\text { Pvt. Responsiv eness Actual }\end{array}$ & .8124 & .7630 & 7.308E-02 & .6675 & .9572 & 11.116 & 108 & .000 \\
\hline Pair 2 & $\begin{array}{l}\text { Pvt. Tangible Expect - Pvt. } \\
\text { Tangible Actual }\end{array}$ & $-3.29 \mathrm{E}-02$ & .7984 & 7.647E-02 & -.1845 & .1187 & -.430 & 108 & .668 \\
\hline Pair 3 & $\begin{array}{l}\text { Pvt. Empathy Expect - Pvt. } \\
\text { Empathy Actual }\end{array}$ & .4909 & .7770 & 7.409E-02 & .3441 & .6377 & 6.626 & 109 & .000 \\
\hline Pair 4 & $\begin{array}{l}\text { Pvt. Reliability Expect - Pvt. } \\
\text { Reliability Actual }\end{array}$ & .6804 & .7752 & $7.425 \mathrm{E}-02$ & .5333 & .8276 & 9.164 & 108 & .000 \\
\hline Pair 5 & $\begin{array}{l}\text { Pvt. Assurance Expect - Pvt. } \\
\text { Assurance Actual }\end{array}$ & .5917 & .8530 & 8.170E-02 & .4298 & .7537 & 7.243 & 108 & .000 \\
\hline
\end{tabular}

\section{Figure 5 - Pvt. Banks' Expectations vs. Actual - Paired Samples Test}

Following is the analysis of the above results -

H1: Null hypothesis is rejected. There is significant difference in all factors (except Tangible) between expectations and actual of private sector banks.

Following is list of factors showing the service quality gap (descending order)

Assurance

Empathy

Reliability

Responsiveness

The service quality gap is lesser as compared to public sector banks (refer next section).

\section{T-Test - Public Banks Expectations vs. Actual}

\section{Paired Samples Statistics}

\begin{tabular}{|ll|r|r|r|r|}
\hline & & Mean & N & Std. Deviation & $\begin{array}{c}\text { Std. Error } \\
\text { Mean }\end{array}$ \\
\hline Pair 1 & Pub. Responsiveness Expect & 5.5400 & 10 & .4719 & .1492 \\
& Pub Responsiveness Actual & 3.2000 & 10 & 1.2754 & .4033 \\
Pair 2 & Pub. Tangible Expect & 4.7500 & .4410 & .1394 \\
& Pub Tangible Actual & 3.1000 & 10 & 10 & .2614 \\
Pair 3 & Pub. Empathy Expect & 5.2000 & 10 & .8266 & .1740 \\
& Pub Empathy Actual & 3.0417 & 10 & .8573 & .2711 \\
Pair 4 & Pub Reliability Expect & 5.5667 & 10 & .5890 & .1863 \\
& Pub Reliability Actual 5 & 3.6000 & 10 & 1.0750 & .3399 \\
& Pub. Assurance Expect & 4.2500 & 10 & 1.5501 & .4902 \\
& Pub Assurance Actual & 2.6500 & 10 & 1.2483 & .3948 \\
\hline
\end{tabular}

Figure 6 - Pub Banks Expectations vs. Actual - Paired Samples Stats

\begin{tabular}{|c|c|c|c|c|c|c|c|c|c|}
\hline \multicolumn{10}{|c|}{ Paired Samples Test } \\
\hline & & \multicolumn{5}{|c|}{ Paired Diff erences } & \multirow[b]{3}{*}{$t$} & \multirow[b]{3}{*}{ df } & \multirow[b]{3}{*}{ Sig. (2-tailed) } \\
\hline & & \multirow[b]{2}{*}{ Mean } & \multirow[b]{2}{*}{ Std. Deviation } & \multirow{2}{*}{$\begin{array}{l}\text { Std. Error } \\
\text { Mean }\end{array}$} & \multicolumn{2}{|c|}{$\begin{array}{l}95 \% \text { Confidence } \\
\text { Interv al of the } \\
\text { Diff erence }\end{array}$} & & & \\
\hline & & & & & Lower & Upper & & & \\
\hline Pair 1 & $\begin{array}{l}\text { Pub. Responsiveness } \\
\text { Expect - Pub } \\
\text { Responsiveness Actual }\end{array}$ & 2.3400 & 1.5693 & .4963 & 1.2174 & 3.4626 & 4.715 & 9 & .001 \\
\hline Pair 2 & $\begin{array}{l}\text { Pub. Tangible Expect - } \\
\text { Pub Tangible Actual }\end{array}$ & 1.6500 & 1.0750 & .3399 & .8810 & 2.4190 & 4.854 & 9 & .001 \\
\hline Pair 3 & $\begin{array}{l}\text { Pub. Empathy Expect - } \\
\text { Pub Empathy Actual }\end{array}$ & 2.1583 & 1.2079 & .3820 & 1.2943 & 3.0224 & 5.650 & 9 & .000 \\
\hline Pair 4 & $\begin{array}{l}\text { Pub Reliability Expect - } \\
\text { Pub Reliability Actual }\end{array}$ & 1.9667 & 1.0824 & .3423 & 1.1924 & 2.7410 & 5.746 & 9 & .000 \\
\hline Pair 5 & $\begin{array}{l}\text { Pub. Assurance Expect } \\
\text { - Pub Assurance Actual }\end{array}$ & 1.6000 & 1.8379 & .5812 & .2853 & 2.9147 & 2.753 & 9 & .022 \\
\hline
\end{tabular}

Figure 7 - Pub Banks Expectations vs. Actual - Paired Samples Test

Following is the analysis of the above results -

H2: Null hypothesis is rejected. There is significant difference in all factors between expectations and actual of public sector banks.

Following is list of factors showing the service quality gap (descending order)

Assurance 
Responsiveness

Empathy

Reliability

Tangible

The service quality gap is more as compared to private sector banks (refer last section).

\section{T-Test - Pvt. Banks Expectations vs. Public Banks Expectations}

\begin{tabular}{|ll|r|r|r|r|}
\multicolumn{1}{|c|}{ Group Statistics } \\
\hline & Bank Ty pe & $N$ & Mean & Std. Dev iation & $\begin{array}{r}\text { Std. Error } \\
\text { Mean }\end{array}$ \\
\hline Responsiv eness Expect & Priv ate & 111 & 5.5306 & .3809 & $3.615 \mathrm{E}-02$ \\
& Public & 10 & 5.5400 & .4719 & .1492 \\
\hline & Priv ate & 111 & 4.7725 & .6954 & $6.600 \mathrm{E}-02$ \\
& Public & 10 & 4.7500 & .4410 & .1394 \\
\hline Tangible Expect & Priv ate & 111 & 5.1269 & .5368 & $5.095 \mathrm{E}-02$ \\
& Public & 10 & 5.2000 & .5503 & .1740 \\
\hline Reliability Expect & Priv ate & 111 & 5.6411 & .4774 & $4.531 \mathrm{E}-02$ \\
& Public & 10 & 5.5667 & .5890 & .1863 \\
\hline Assurance Expect & Priv ate & 111 & 5.0946 & .6726 & $6.384 \mathrm{E}-02$ \\
& Public & 10 & 4.2500 & 1.5501 & .4902 \\
\hline
\end{tabular}

Figure 8 - Pvt. Banks Expectations vs. Public Banks Expectations - Group Stats

\begin{tabular}{|c|c|c|c|c|c|c|c|c|c|c|}
\hline \multicolumn{11}{|c|}{ Independent Samples Test } \\
\hline & & \multicolumn{2}{|c|}{$\begin{array}{l}\text { Levene's Test for } \\
\text { Equality of Variances }\end{array}$} & \multicolumn{7}{|c|}{ t-test for Equality of Means } \\
\hline & & \multirow[b]{2}{*}{$\mathrm{F}$} & \multirow[b]{2}{*}{ Sig. } & \multirow[b]{2}{*}{$t$} & \multirow[b]{2}{*}{$\mathrm{df}$} & \multirow[b]{2}{*}{ Sig. (2-tailed) } & \multirow{2}{*}{$\begin{array}{c}\text { Mean } \\
\text { Diff ference }\end{array}$} & \multirow{2}{*}{$\begin{array}{l}\text { Std. Error } \\
\text { Diff ference }\end{array}$} & \multicolumn{2}{|c|}{$\begin{array}{l}95 \% \text { Confidence } \\
\text { Interv al of the } \\
\text { Diff erence }\end{array}$} \\
\hline & & & & & & & & & Lower & Upper \\
\hline \multirow[t]{2}{*}{ Responsiveness Expect } & $\begin{array}{l}\text { Equal variances } \\
\text { assumed }\end{array}$ & 1.246 & .267 & -.073 & 119 & .942 & $-9.369 \mathrm{E}-03$ & .1283 & -.2633 & .2446 \\
\hline & $\begin{array}{l}\text { Equal variances } \\
\text { not assumed }\end{array}$ & & & -.061 & 10.085 & .953 & $-9.369 \mathrm{E}-03$ & .1535 & -.3511 & .3323 \\
\hline \multirow[t]{2}{*}{ Tangible Expect } & $\begin{array}{l}\text { Equal variances } \\
\text { assumed }\end{array}$ & 2.649 & .106 & .100 & 119 & .920 & $2.252 \mathrm{E}-02$ & .2243 & -.4217 & .4667 \\
\hline & $\begin{array}{l}\text { Equal variances } \\
\text { not assumed }\end{array}$ & & & .146 & 13.430 & .886 & $2.252 \mathrm{E}-02$ & .1543 & -.3097 & .3547 \\
\hline \multirow[t]{2}{*}{ Empathy Expect } & $\begin{array}{l}\text { Equal variances } \\
\text { assumed }\end{array}$ & .038 & .847 & -.412 & 119 & .681 & $-7.312 \mathrm{E}-02$ & .1776 & -.4247 & .2785 \\
\hline & $\begin{array}{l}\text { Equal variances } \\
\text { not assumed }\end{array}$ & & & -.403 & 10.603 & .695 & $-7.312 \mathrm{E}-02$ & .1813 & -.4740 & .3278 \\
\hline \multirow[t]{2}{*}{ Reliability Expect } & $\begin{array}{l}\text { Equal variances } \\
\text { assumed }\end{array}$ & 1.204 & .275 & .463 & 119 & .644 & 7.447E-02 & .1607 & -.2437 & .3927 \\
\hline & $\begin{array}{l}\text { Equal variances } \\
\text { not assumed }\end{array}$ & & & .389 & 10.094 & .706 & 7.447E-02 & .1917 & -.3521 & .5010 \\
\hline \multirow[t]{2}{*}{ Assurance Expect } & $\begin{array}{l}\text { Equal variances } \\
\text { assumed }\end{array}$ & 15.243 & .000 & 3.303 & 119 & .001 & .8446 & .2557 & .3383 & 1.3509 \\
\hline & $\begin{array}{l}\text { Equal variances } \\
\text { not assumed }\end{array}$ & & & 1.709 & 9.308 & .121 & .8446 & .4943 & -.2680 & 1.9572 \\
\hline
\end{tabular}

Figure 9 - Pvt. Banks Expectations vs. Public Banks Expectations - Independent Samples Test

Following is the analysis of the above results -

H3: Null hypothesis is accepted. There is no significant difference between consumers' expectations from private sector banks and private sector banks.

\section{T-Test - Pvt. Banks Actual vs. Public Banks Actual}

Group Statistics

\begin{tabular}{|c|c|c|c|c|c|}
\hline & Bank Type & $\mathbf{N}$ & Mean & Std. Dev iation & $\begin{array}{c}\text { Std. Error } \\
\text { Mean }\end{array}$ \\
\hline \multirow[t]{2}{*}{ Responsiv eness Actual } & Priv ate & 109 & 4.7243 & .6852 & $6.563 E-02$ \\
\hline & Public & 10 & 3.2000 & 1.2754 & .4033 \\
\hline \multirow[t]{2}{*}{ Tangible Actual } & Priv ate & 109 & 4.7943 & .6097 & 5.840E-02 \\
\hline & Public & 10 & 3.1000 & .8266 & .2614 \\
\hline \multirow[t]{2}{*}{ Empathy Actual } & Priv ate & 110 & 4.6280 & .6466 & $6.166 E-02$ \\
\hline & Public & 10 & 3.0417 & .8573 & .2711 \\
\hline \multirow[t]{2}{*}{ Reliability Actual } & Priv ate & 109 & 4.9664 & .6510 & $6.235 \mathrm{E}-\mathrm{O} 2$ \\
\hline & Public & 10 & 3.6000 & 1.0750 & .3399 \\
\hline \multirow[t]{2}{*}{ Assurance Actual } & Priv ate & 109 & 4.5183 & .7933 & $7.598 \mathrm{E}-\mathrm{O} 2$ \\
\hline & Public & 10 & 2.6500 & 1.2483 & .3948 \\
\hline
\end{tabular}

Figure 10 - Pvt. Banks Actual vs. Pub Banks Actual - Group Stats 


\begin{tabular}{|c|c|c|c|c|c|c|c|c|c|c|}
\hline \multicolumn{11}{|c|}{ Independent Samples Test } \\
\hline & & \multicolumn{2}{|c|}{$\begin{array}{l}\text { Levene's Test for } \\
\text { Equality of Variances }\end{array}$} & \multicolumn{7}{|c|}{ t-test for Equality of Means } \\
\hline & & \multirow[b]{2}{*}{$\mathrm{F}$} & \multirow[b]{2}{*}{ Sig. } & \multirow[b]{2}{*}{$t$} & \multirow[b]{2}{*}{ df } & \multirow[b]{2}{*}{ Sig. (2-tailed) } & \multirow{2}{*}{$\begin{array}{c}\text { Mean } \\
\text { Difference }\end{array}$} & \multirow{2}{*}{$\begin{array}{l}\text { Std. Error } \\
\text { Difference }\end{array}$} & \multicolumn{2}{|c|}{$\begin{array}{l}95 \% \text { Confidence } \\
\text { Interv al of the } \\
\text { Diff erence }\end{array}$} \\
\hline & & & & & & & & & Lower & Upper \\
\hline \multirow[t]{2}{*}{ Responsiveness Actual } & $\begin{array}{l}\text { Equal variances } \\
\text { assumed }\end{array}$ & 11.597 & .001 & 6.173 & 117 & .000 & 1.5243 & .2469 & 1.0353 & 2.0134 \\
\hline & $\begin{array}{l}\text { Equal variances } \\
\text { not assumed }\end{array}$ & & & 3.730 & 9.482 & .004 & 1.5243 & .4086 & .6071 & 2.4416 \\
\hline \multirow[t]{2}{*}{ Tangible Actual } & $\begin{array}{l}\text { Equal variances } \\
\text { assumed }\end{array}$ & 2.515 & .115 & 8.152 & 117 & .000 & 1.6943 & .2078 & 1.2827 & 2.1060 \\
\hline & $\begin{array}{l}\text { Equal variances } \\
\text { not assumed }\end{array}$ & & & 6.326 & 9.919 & .000 & 1.6943 & .2679 & 1.0969 & 2.2918 \\
\hline \multirow[t]{2}{*}{ Empathy Actual } & $\begin{array}{l}\text { Equal variances } \\
\text { assumed }\end{array}$ & 2.008 & .159 & 7.222 & 118 & .000 & 1.5864 & .2197 & 1.1514 & 2.0214 \\
\hline & $\begin{array}{l}\text { Equal variances } \\
\text { not assumed }\end{array}$ & & & 5.706 & 9.953 & .000 & 1.5864 & .2780 & .9665 & 2.2062 \\
\hline \multirow[t]{2}{*}{ Reliability Actual } & $\begin{array}{l}\text { Equal variances } \\
\text { assumed }\end{array}$ & 10.020 & .002 & 5.968 & 117 & .000 & 1.3664 & .2289 & .9130 & 1.8197 \\
\hline & $\begin{array}{l}\text { Equal variances } \\
\text { not assumed }\end{array}$ & & & 3.954 & 9.615 & .003 & 1.3664 & .3456 & .5921 & 2.1406 \\
\hline \multirow[t]{2}{*}{ Assurance Actual } & $\begin{array}{l}\text { Equal variances } \\
\text { assumed }\end{array}$ & 5.437 & .021 & 6.755 & 117 & .000 & 1.8683 & .2766 & 1.3206 & 2.4161 \\
\hline & $\begin{array}{l}\text { Equal variances } \\
\text { not assumed }\end{array}$ & & & 4.648 & 9.678 & .001 & 1.8683 & .4020 & .9686 & 2.7681 \\
\hline
\end{tabular}

\section{Figure 11 - Pvt. Banks Actual vs. Pub Banks Actual - Independent Samples Test}

Following is the analysis of the above results -

H4: Null hypothesis is rejected. There is significant difference in all factors between private sector banks actual offerings versus public sector banks actual offerings.

\section{Findings -}

Following are the key findings of this study -

There is not any significant difference between consumers' expectations between private and public sector banks. There is significant difference between private and public sector banks' actual offerings.

There is significant difference (except Tangible factor) between consumers' expectations and bank's actual offerings for Private sector banks. There is significant difference between consumers' expectations and bank's actual offerings for Public sector banks. The deviation of consumers' expectations and bank's actual offerings is more in public sector banks as compared to private sector banks.

\section{Conclusions:}

There is significant difference in consumers' expectations from their private sector banks and the extent to which their bank is engaged in the above practices.

There is significant difference in consumers' expectations from their public sector banks and the extent to which their bank is engaged in the above practices.

There is no significant difference in consumers' expectations from private sector and public sector banks.

There is significant difference in the private and public sector banks actual offerings to their consumers.

Public sector banks has more gap in expectations vs. actual as compared to private sector banks.

Following are the factors showing the significant service quality gap in private sector banks -

Assurance, empathy, reliability, responsiveness.

Following are the factors showing the significant service quality gap in public sector banks -

Assurance, Responsiveness, Empathy, Reliability, Tangible

\section{References:}

[1]. Report on Trend and Progress of Banking in India 1998-99, Reserve Bank of India, Supplement to RBI Bulletin, December 1999.

[2]. Report on Trend $=$ and Progress of Banking in India 1997-98, Reserve

[3]. Bank of India, Supplement to RBI Bulletin, = November 1998.

[4]. Reserve Bank of India Annual Report 1998-99, Supplement to RBI Bulletin, September 1999.

[5]. "Service Quality Gap and Customers' Satisfactions of Commercial Banks in Malaysia" by Izaz Mohd Tahir and Nor Mazlina Abu Bakar.

[6]. The dimensions of service quality: A study of Indian retail banking environment Customer expectations and perceptions across the Indian banking industry and the resultant financial implications by Dutta, Kirti and Dutta, Anil. 